\title{
Closing the Cold CNO Cycle: A New Measurement of ${ }^{19} \mathbf{F}(\mathbf{p}, \gamma)$
}

\section{Aaron Couture*}

Los Alamos National Laboratory

E-mail: acoutureelanl.gov

\section{Manoël Couder}

University of Notre Dame

E-mail: mcouderend.edu

\section{Joachim Görres}

University of Notre Dame

E-mail: jgoerresand.edu

\section{Hye Young Lee}

University of Notre Dame

E-mail: hlee2@nd.edu

\section{Edward Stech}

University of Notre Dame

E-mail: estechand.edu

\section{Elizabeth Strandberg}

University of Notre Dame

E-mail: emcnassaend.edu

\section{Wanpeng Tan}

University of Notre Dame

E-mail: wtanend.edu

\section{Claudio Ugalde}

University of North Carolina, Chapel Hill

E-mail: cugalde@unc.edu

\section{Michael Wiescher}

University of Notre Dame

E-mail: wiescher.1@nd.edu 
The cold CNO cycle is the primary energy source for main sequence hydrogen burning in intermediate to heavy mass stars $\left(\mathrm{M}>1.5 \mathrm{M}_{\odot}\right)$. The ${ }^{19} \mathrm{~F}(\mathrm{p}, \gamma)$ reaction provides the only breakout path during quiescent hydrogen burning, and the cyclic nature of the process enhances small breakout components. The sign of the interference components provided the largest uncertainty in the reaction rate since no measurements had been sufficiently sensitive to measure the reaction off-resonance. A new detection technique developed at the University of Notre Dame allowed a measurement of the ${ }^{19} \mathrm{~F}(\mathrm{p}, \gamma)$ and ${ }^{19} \mathrm{~F}\left(\mathrm{p}, \alpha_{i} \gamma\right)$ reactions over an energy range of $\mathrm{E}_{p}=200-800 \mathrm{keV}$. This allowed a new measurement of the resonance parameters as well as a first measurement of the signs of the interference terms. Partial widths and resonance strengths are reported for the resonances in this region. 


\section{Introduction}

The cold CNO cycle is a catalytic hydrogen burning mechanism, using carbon, nitrogen, and oxygen seed material in the hydrogen burning core of a main-sequence star. In order for the CNO cycle to compete with the primary p-p chain reactions, the temperatures must exceed $20 \mathrm{MK}$, corresponding to stars of $1.5 \mathrm{M}_{\odot}$ or greater mass [1]. Since the reactions involved in the cold CNO cycle, illustrated in figure 1, are taking place at relatively low temperature, no nucleosynthesis beyond the conversion of hydrogen to helium is possible. The CNO material comes solely from the proto-stellar material that condensed to form the star. The only reaction which can potentially remove the catalytic material from the cycle is ${ }^{19} \mathrm{~F}(\mathrm{p}, \gamma){ }^{20} \mathrm{Ne}$ [泡]. This reaction was expected to be rather weak compared to the the ${ }^{19} \mathrm{~F}(\mathrm{p}, \alpha){ }^{16} \mathrm{O}$ reaction, so that the $\mathrm{CNO}$ material would be recycled back to ${ }^{16} \mathrm{O}$, with no material breaking out to ${ }^{20} \mathrm{Ne}$.

Unfortunately, the strength of the gamma-producing ${ }^{19} \mathrm{~F}(\mathrm{p}, \alpha \gamma)$ reactions made measurements of the ${ }^{19} \mathrm{~F}(\mathrm{p}, \gamma)$ cross-section quite difficult. Previous measurements relied on detecting the $>11 \mathrm{MeV}$ primary transition to the first excited state of ${ }^{20} \mathrm{Ne}$ [3]-77. Most of these prior measurements relied on low resolution and relatively small volume $\mathrm{NaI}(\mathrm{Tl})$ detectors. This made all of the measurements very susceptible to mis-identification of pile-up from the $6.1 \mathrm{MeV}$ gamma-rays from the competing $\alpha-\gamma$ reaction as the energy resolution was insufficient to separate the two components. Subotić [7] was the only measurement which employed a high-resolution $\mathrm{Ge}(\mathrm{Li})$ detector, though due to the low detection efficiency for such high energy gamma-rays, the measurement was limited to observing on-resonance components 가. Unsurprisingly, there was discrepancy between the different measurements. One measurement observed an anomalously high partial width for the broad resonance at $\mathrm{E}_{\mathrm{cm}}=567 \mathrm{keV}$ [6]. None of the measurements were sufficiently sensitive to determine the signs of the interference components which were expected to contribute almost $50 \%$ to the reaction rate at stellar temperatures [8, 9 .

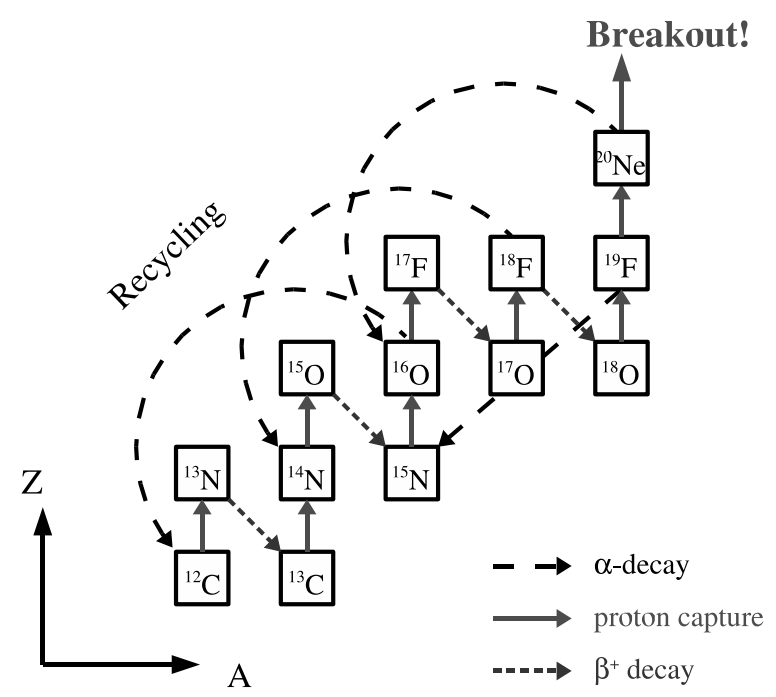

Figure 1: The CNO cycle operating a temperatures below $\mathrm{T}_{6}=100$ 


\section{Experimental Procedure}

\subsection{Beam and Target Production}

All of the present measurements were made at the University of Notre Dame Nuclear Structure Laboratory. The JN model Van de Graaff accelerator was used to produce proton beams of energies from 200 to $700 \mathrm{keV}$ lab. The $\mathrm{KN}$ accelerator was used for the region from 600 to $800 \mathrm{keV}$, ensuring a broad overlap region to ensure the results were consistent. All of the experiments were carried out on the same beamline, with only the accelerator providing beam to the target changing.

The targets were prepared by evaporating $\mathrm{CaF}_{2}$ powder on to tantalum backings. Since beamstop targets were used, the charge was collected on target and used to determine the total number of protons. A biased cold finger was used both to prevent carbon build-up and ensure reliable charge collection. Degradation of the fluorine was observed in the target and corrections were made for this effect. Two different target thicknesses were used to confirm the results observed in the inter-resonance regions. Reference [10] contains further details of the target preparation and the corrections for target degradation.

\subsection{Detection Array}

Significant advances have been made in detector technologies since the previous measurements had been made, making available both large volume HPGe detectors and $\mathrm{NaI}(\mathrm{Tl})$ detectors. To take full advantage of this, a hybrid detection system was designed which could exploit the high efficiency of large volume $\mathrm{NaI}(\mathrm{Tl})$ detectors with the high resolution offered by HPGe detectors. Four 8" diameter and 6" tall cylindrical $\mathrm{NaI}(\mathrm{Tl})$ detectors were used together with a $150 \%$ HPGe clover detector. The detectors were arranged as is shown in figure 2. The HPGe detector was positioned directly in front of the target holder, mounted at $0^{\circ}$ in order to cover the largest possible solid angle. The segmentation of the HPGe clover allowed an instantaneous count rate in each crystal of as much as $15 \mathrm{kHz}$. The four $\mathrm{NaI}(\mathrm{Tl})$ detectors were mounted at backward angles and rotated $45^{\circ}$ in $\phi$ and $\theta$ in order to offer an improved solid angle coverage and to maximize the detector depth as seen from the target.

The idea of the setup was to observe the $1.63 \mathrm{MeV}$ transition from the first excited state to the ground state in the HPGe detector while observing the primary transition in the $\mathrm{NaI}(\mathrm{Tl})$ detectors. A coincidence requirement was placed between the $\mathrm{NaI}(\mathrm{Tl})$ detectors and the HPGe crystals. In the event of a coincidence, a sum energy gate was placed above $10 \mathrm{MeV}$. Since the Q-value was 12.484 MeV, this gate was high enough to filter out most of the 6-7 MeV lines from the (p, $\alpha \gamma$ ) reaction while keeping the coincidence efficiency fairly insensitive to the incident beam energy. If both conditions were met, then the $1.63 \mathrm{MeV}$ gamma yield was measured in the HPGe clover. By requiring the coincidence in hardware, it was possible to run at rates of $10-12 \mathrm{kHz}$ in the individual HPGe detectors while still only requiring acquisition at a rate of $4 \mathrm{kHz}$, keeping the live time well above $80 \%$. The effectiveness of these gating conditions can be seen in figure 3. At the same time, one of every twenty events in the germanium detector was passed through to the acquisition regardless of coincidence so that the (p, $\alpha \gamma)$ yield could be monitored simultaneously with the $(p, \gamma)$. These two features proved to be essential as it both gave us rapid feedback on the energy and resonance structure as well as allowed running at high beam currents to see the $(p, \gamma)$ yield in a reasonable amount of time. The coincidence requirement suppressed the detection 


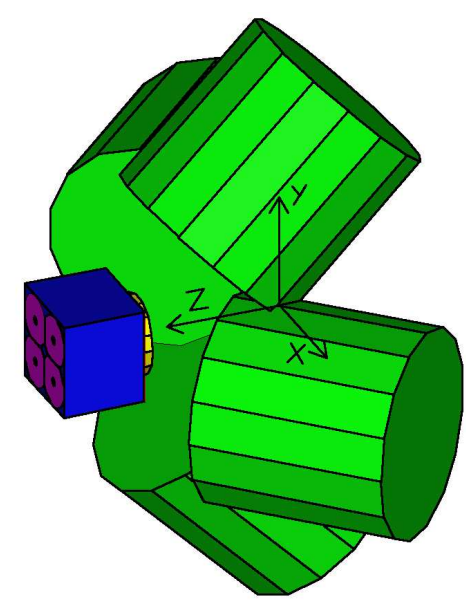

Figure 2: A schematic of the detector array used is shown above. The HPGe detector is on left, centered and mounted at $0^{\circ}$ with respect to the beam. The NaI(Tl) detectors are primarily at backward angles, rotated to provide maximum solid angle coverage. The beam path was collinear with the positive $\mathrm{z}$-axis.

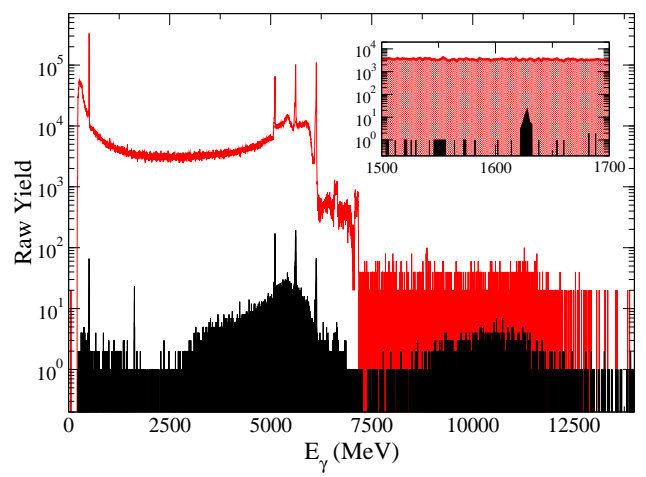

Figure 3: The suppression of the (p, $\alpha \gamma)$ components by the array is shown above. In red (light) is shown the yield seen by the HPGe clover with no cuts or conditions. In black (dark) is the yield after the multiplicity and Q-value cuts. The inset shows the region surrounding the $1.63 \mathrm{MeV}$ first excited state decay of ${ }^{20} \mathrm{Ne}$ in greater detail. A suppression of close to $10^{4}$ was achieved.

of transitions directly to the ground state as these transitions only exhibit a single gamma ray. Previous measurements had not observed any significant yield from ground state transitions. In order to monitor any unexpected yield in this channel, signals in the HPGe detector with greater than $8 \mathrm{MeV}$ of energy were also recorded. The acquisition was designed to allow the separation of events from the different trigger types (coincidence, singles, and high energy) when the data was analyzed. No significant yield was seen in the HPGe detector above $\approx 11.5 \mathrm{MeV}$, an energy corresponding to a transition from the resonant state to the first excited state. Signals above $12 \mathrm{MeV}$ would have indicated that there was significant decay directly to the ground state which would warrant closer inspection.

\section{Analysis and Results}

Experimental yield curves were measured for the (p, $\left.\gamma_{1}\right),\left(\mathrm{p}, \alpha_{2}\right),\left(\mathrm{p}, \alpha_{3}\right)$, and $\left(\mathrm{p}, \alpha_{4}\right)$ channels. The (p, $\left.\gamma_{2}\right)$ was also monitored, but no significant yield was observed outside of the $\mathrm{E}_{\mathrm{cm}}=633$ $\mathrm{keV}$ resonance. The resonance energies, spins, and parities are well known [11], so no effort was made to measure them. The yield curves were fit with a set of Breit-Wigner resonance shapes. Interference terms were calculated for the resonances. The total yield was calculated by

$$
Y=\sum_{i}\left(\int_{E-\chi}^{E} \frac{\sigma_{i}(E)}{\varepsilon(E)} \mathrm{d} E\right)+\sum_{i<j}\left(\delta\left(J_{i}^{\pi}, J_{j}^{\pi}\right) \int_{E-\chi}^{E} \frac{ \pm 2\left(\sigma_{i} \sigma_{j}\right)^{\frac{1}{2}}}{\varepsilon(E)}\left(\cos \left(\varphi_{R_{i}}-\varphi_{R_{j}}\right) \mathrm{d} E\right) .\right.
$$

The sum is over resonances $i$, the cross-sections were Breit-Wigner cross-sections [12], $\chi$ is the target thickness in terms of energy, $\varepsilon(E)$ is the energy loss of the beam, $J_{i}^{\pi}$ is the spin and parity of 


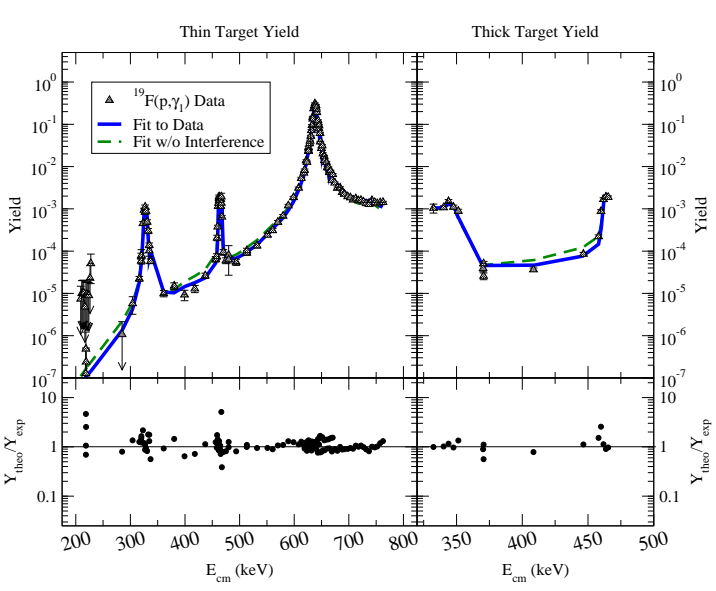

Figure 4: The fit of the (p, $\left.\gamma_{1}\right)$ data is shown above. The agreement between the data and the fit is quite good. On the panel on the right is shown the fit to the thick target data. Note the enhanced sensitivity to the interference component. The ratio of the calculated yield to the observed yield is shown at the bottom.

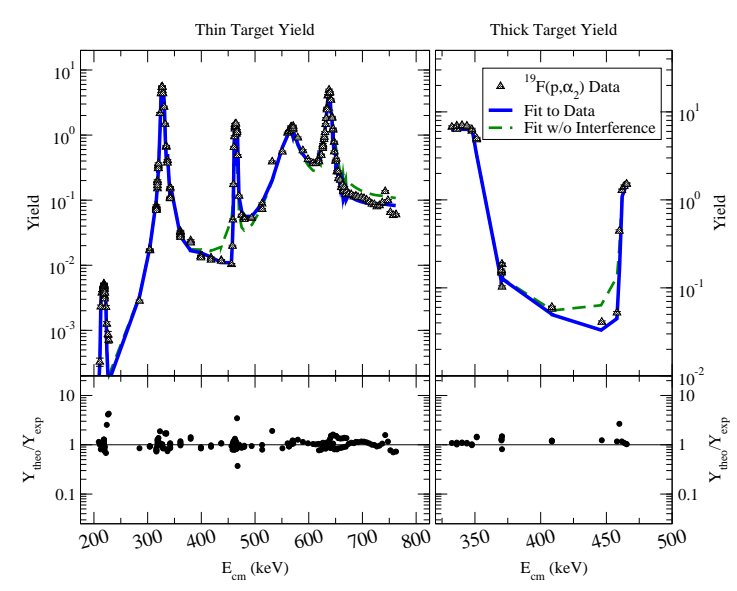

Figure 5: The fit of the (p, $\left.\alpha_{2}\right)$ data is shown above. The acquisition method allowed the $\alpha$ channel data to be taken simultaneously with the $\gamma$ channel data which was of significant advantage in the fitting of the data and improved systematics. Again, the thick target yield and fit are shown in the panel on the right.

the resonant state, and $\varphi_{R}$ is the resonance phase shift [13]. For total yield calculations, only terms with the same $J^{\pi}$ exhibit interference effects; the $\delta$ function performs this selection. It is worth noting that since the phase shift is defined as an arctan function, care must be taken in choosing the appropriate range so that the cosine function behaves properly. The numerical integration was completed with the dgauss routine from the cernlib package [14]. The resonance parameters were allowed to vary freely and minimized with the minuit minimization package [15]. The sign of the interference term, which has to be determined experimentally, was changed manually in order to find the optimal fit. All four reaction channels were fit simultaneously. The total width was calculated from the partial widths and thus, varied, though not independently from the physical constraints. A background state to simulate the contributions of all higher lying resonances was included, though it only contributed significantly in the $(p, \gamma)$ yield. Fits to two of the yield curves can be seen in figures $甘$ and 5. As can be seen, the yield is well reproduced. The resonance parameters are given in table 1. While previous measurements were generally normalized to the strength of $669 \mathrm{keV}$ resonance, in this measurement the absolute efficiency of the HPGe clover was determined both by use of a ${ }^{60} \mathrm{Co}$ source as well as looking at decays from the $12.239 \mathrm{MeV}$ state of ${ }^{28} \mathrm{Si}$ populated via the ${ }^{27} \mathrm{Al}(\mathrm{p}, \gamma)$ reaction. The resonance strength was taken from ref. [16], while the branching ratios were taken from ref. [17]. The state was chosen because the energies of the gamma-decays were similar to the gamma-ray energies from the decay of the states in ${ }^{20} \mathrm{Ne}^{*}$ and ${ }^{16} \mathrm{O}^{*}$. Summing corrections were accounted for in the determination of the absolute efficiency [10]. The determination of the coincidence efficiency was made by comparing the singles yield to the coincidence yield for the $669 \mathrm{keV}$ resonance in ${ }^{19} \mathrm{~F}(\mathrm{p}, \gamma)$. GEANT [18] simulations were done to determine any change in coincidence efficiency due to the incident proton energy. The correction due to proton energy was less than $5 \%$ at lowest proton energies. 


\begin{tabular}{|c|c|c|c|c|c|}
\hline \multirow{2}{*}{$\begin{array}{l}\text { Author } \\
\text { Quantity }\end{array}$} & \multicolumn{5}{|c|}{ Energy, cm (keV) } \\
\hline & 212 & 323 & 460 & 567 & 636 \\
\hline $\mathrm{J}^{\pi}[11]$ & $2^{-}$ & $1^{+}$ & $1^{+}$ & $2^{-}$ & $1^{+}$ \\
\hline \multicolumn{6}{|l|}{$\begin{array}{c}\text { This } \\
\text { Measurement }\end{array}$} \\
\hline$\Gamma_{p} \quad(\mathrm{eV})$ & 0.011 & 35.9 & 12.2 & 44.5 & 6540 \\
\hline$(\mathrm{eV})$ & n.o. & 0.096 & 0.248 & n.o. & 1.28 \\
\hline$(\mathrm{eV})$ & 861 & 1960 & 753 & 35800 & 71.2 \\
\hline$(\mathrm{keV})$ & 870 & 2060 & 1060 & 36100 & 6680 \\
\hline$(\omega \gamma)_{\gamma_{1}} \quad(\mathrm{meV})$ & n.o. & $1.25 \times 10^{-3}$ & $2.14 \times 10^{-3}$ & n.o. & 940 \\
\hline$\sigma_{R} \quad(\mu \mathrm{b})$ & n.o. & 0.51 & 1.2 & n.o. & 610 \\
\hline \multicolumn{6}{|l|}{ Subotić [四] } \\
\hline $\begin{array}{ll}\Gamma_{\gamma_{1}} & (\mathrm{eV})\end{array}$ & - & 0.80 & 0.59 & 3.93 & - \\
\hline$(\omega \gamma)_{\gamma_{1}} \quad(\mathrm{meV})$ & 一 & 0.01 & 0.0025 & 0.0053 & 一 \\
\hline \multicolumn{6}{|l|}{ Berkes [6] } \\
\hline $\begin{array}{ll}\Gamma_{p} & (\mathrm{eV})\end{array}$ & $\approx 0.43$ & - & 4.8 & - & - \\
\hline$(\mathrm{eV})$ & $<0.06$ & 0.27 & 0.40 & 11.4 & - \\
\hline$(\mathrm{eV})$ & 950 & - & 670 & - & 一 \\
\hline \multicolumn{6}{|l|}{ Sinclair [3] } \\
\hline$\sigma_{R} \quad(\mu \mathrm{b})$ & - & 一 & - & - & 480 \\
\hline
\end{tabular}

Table 1: Note that all widths and strengths are in center of mass frame. For those entries where "- is listed, the relevant parameter was not measured by that experiment. Those resonances with a value of "n.o." for this measurement were not observed. The strength was sufficiently small as to be below the limits of detection. It is worth noting that the values observed by Subotić and Berkes for the $567 \mathrm{keV}$ resonance were well within the experimental sensitivity.

\section{Conclusions}

The new measurements of the resonance parameters and most importantly, the interference terms, is quite promising. In order to properly fit the high energy tail of the $633 \mathrm{keV}$ resonance, it was necessary to include a broad, high energy $1^{+}$state to account for the contribution of all higher lying resonances. Unfortunately, the properties of this background state were not well constrained by the present data, and while these uncertainties had little effect on the resonance properties in the region studied, they are sufficient to cause large uncertainties on the S-factor at astrophysically interesting regions (30-120 keV). Measurements at higher energies, particularly of the possible $\mathrm{T}=1$ resonance at $\mathrm{E}_{\mathrm{cm}}=1.35 \mathrm{MeV}$, [11] are needed to constrain this background term.

The measurement technique has shown to be very effective at isolating the ${ }^{19} \mathrm{~F}(\mathrm{p}, \gamma)$ reaction and is sufficiently sensitive to determine interference terms, even in regions of resonances in the competing channels. There was no observed strength for the lowest-lying resonance at 
$\mathrm{E}_{\mathrm{cm}}=212 \mathrm{keV}$. The broad resonance observed most clearly by [6] at $\mathrm{E}_{\mathrm{cm}}=567 \mathrm{keV}$ is excluded so that it will not contribute in any significant way to the astrophysical reaction rate. The resonance strengths for the other resonances were generally smaller than that previously observed, and the net interference effect at low energies was seen to be destructive. All of these effects suggest that the reaction rate will be lower than previously predicted, effectively closing the cold CNO cycle. A final determination of the stellar reaction rate and $\mathrm{S}$-factor will require further measurements at higher energies to constrain the role of the background state needed to properly fit the data.

\section{Acknowledgments}

The authors would like to thank Dr. Larry Lamm, Mr. Bradley Mulder, Mr. James Kaiser, and Mr. Jerry Lingle for their technical assistance with the maintenance and operation of the accelerators at the Nuclear Structure Laboratory at the University of Notre Dame.

This work was supported by the National Science Foundation under Grant No.

PHY01-40324, the Joint Institute for Nuclear Astrophysics, NSF-PFC, under Grant No.

PHY02-16783 and the US Department of Energy under the auspices of Los Alamos National Security, LLC, DOE contract number DE-AC52-06NA25396.

\section{References}

[1] C. Rolfs, W. Rodney. Cauldrons in the Cosmos. University of Chicago Press, Chicago (1988).

[2] M. Wiescher, J. Görres, H. Schatz. Break-out reactions from the CNO cycles. J. Phys. G: Nucl. Part. Phys. 25 (1999) R133.

[3] R. M. Sinclair. Gamma Radiation from Certain Nuclear Reactions. Phys. Rev. 93 (1954) 1082.

[4] G. K. Farney, H. H. Given, B. D. Kern, H. T. M. High Energy Gamma Rays from the Proton Bombardment of Fluorine. Physical Review 97 (1955) 720.

[5] L. Keszthelyi, I. Berkes, I. Demeter, I. Fodor. Resonances in ${ }^{19} \mathrm{~F}+p$ Reactions at 224 and $340 \mathrm{keV}$ Proton Energies. Nucl. Phys. 29 (1962) 241.

[6] I. Berkes, I. Dézsi, I. Fodor, L. Keszthelyi. The Resonances at 483 and $597 \mathrm{keV}$ Proton Energies in $F^{19}+$ p Reactions. Nucl. Phys. 43 (1963) 103.

[7] K. M. Subotić, R. Ostojić, B. Z. Stepančić. Study of the ${ }^{19} F(p, \gamma)^{20}$ Ne Radiative Capture Reaction from 0.2-1.2 MeV. Nuclear Physics A 331 (1979) 491.

[8] G. R. Caughlan, W. A. Fowler. Thermonuclear Reaction Rates V. Atomic Data and Nuclear Data Tables 40 (1988) 283.

[9] C. Angulo, M. Arnould, M. Rayet, P. Descouvemont, C. Leclercq-Willain, et al. A compilation of charged-particle induced thermonuclear reaction rates. Nuclear Physics A 656 (1999) 3.

[10] A. Couture. ${ }^{19} F(p, \gamma)^{20}$ Ne and the Stellar CNO Burning Cycle. Ph.D. thesis, University of Notre Dame (2005).

[11] F. Ajzenberg-Selove. Energy levels of light nuclei A = 18-20. Nucl. Phys. A 475 (1987) 1.

[12] H. E. Gove. Resonance Reactions, Experimental. In P. M. Endt, M. Demeur, eds., Nuclear Reactions, vol. 1, chap. 6. Interscience (1959), 259-317.

[13] A. J. Ferguson. Angular Correlation Methods in Gamma-Ray Spectroscopy. North-Holland (1965). 
[14] CERN Development Team (2003).

[15] Application Software Group. MINUIT-Function Minimization and Error Analysis. CERN, Geneva, Switzerland (1998). CERN Program Library Long Writeup D506.

[16] P. B. Lyons, J. W. Toevs, D. G. Sargood. Total Yield Measurements in ${ }^{27} A l(p, \gamma)^{28}$ Si. Nucl. Phys. A 130 (1969) 1.

[17] M. A. Meyer, I. Venter, D. Reitmanm. Energy Levels of ${ }^{28}$ Si. Nucl. Phys. A 250 (1975) 235.

[18] Application Software Group. GEANT - Detector Description and Simulation Tool. CERN, Geneva, Switzerland (1993). CERN Program Library Long Writeup W5013. 